\title{
In Quest of the Best Theoretical Description of Excess Molar Functions of Binary Mixtures of Alcohols
}

\author{
M. Geppert-Rybczyńska ${ }^{a}$ And T. Hofman ${ }^{b}$ \\ ${ }^{a}$ Institute of Chemistry, University of Silesia, Szkolna 7, 40-006 Katowice, Poland \\ ${ }^{b}$ Division of Physical Chemistry, Faculty of Chemistry \\ Warsaw University of Technology \\ Noakowskiego 3, 00-664 Warszawa, Poland \\ An attempt to describe excess molar functions of alkane + alcohol and \\ alcohol + alcohol binary mixtures was made using the theoretical approach \\ constructed on the basis of S-ERAS model and the chemical theory proposed \\ by Campbell. The results obtained seem to be promising, however in order \\ to complete the work, the procedure of determination of fitting parameters as \\ well as relative magnitude of physical and chemical contributions to regarded \\ excess molar quantities must be established.
}

PACS numbers: 05.70.--a, 05.70.Ce, 82.60.Lf

\section{Introduction}

The aim of this work is a presentation of some ideas concerning the description of excess molar functions: excess molar enthalpy, $H^{\mathrm{E}}$, and excess molar volume, $V^{\mathrm{E}}$, of binary mixtures containing one or more alcohols. These compounds are able to associate in the pure state and in the mixture creating "pseudo-chemical" species of a different size and structure. Thus, the main problem in the modeling the properties of such mixtures is a selection of the proper association model which is a base for the construction of the chemical part in any excess molar function.

At the starting point, the most suitable seems to be the extended real associated solution (ERAS) (or symmetrical-ERAS — S-ERAS) model which has been commonly used to description of excess molar functions of the binary mixtures in which different modes of association can occur [1, 2]. The physical part of the ERAS (and S-ERAS) model is based on the Flory theory; next, the chemical contribution assumes the consecutive linear association for alcohols in the pure state and in the mixture. The linear association model postulates an occurrence of only 
linear species with no more than one mixed hydrogen bond (i.e. of the A-B type) per each mixed associate, which strongly disagrees with reality. For this reason the original ERAS (or S-ERAS) model cannot be directly used for binary mixtures of alcohols.

It is also worth of notice that the recent studies based on the application of the ERAS model with the simultaneous description of the Kirkwood factor $\left(g_{\mathrm{K}}\right)$, $H^{\mathrm{E}}$, and IR data for alcohol + cyclohexane mixtures revealed the existence of the few kinds of associated species in such solutions, including the cyclic ones [3]. Thus, the differentiation of the association constants in the chemical part of the model may be one of the most promising way to the correct description of excess molar functions of binary mixtures containing one or two alcohols.

However, in this work, in order to find the solution we postulate counting the number of associated and free as well as bonded mers rather than to define all the possible associates existing in the solution. This approach re-elaborates an idea which was first proposed by Campbell in his chemical theory [4], where the idea of stoichiometric (given by stoichiometry) and true species (the collection of associated complexes existing in solution) was introduced.

Using the assumption given above the chemical contribution to the excess molar volumes and excess molar enthalpies may be expressed by the concentration of free and bounded segments (or bonds) which is an equivalent to the suggestion given above, but makes all calculations simpler. The new formulae were adapted to the description of excess molar volumes and excess molar enthalpies of one alcohol + alkane and two alcohol + alcohol mixtures. The results obtained and some comments will be presented in the next part of this work.

\section{Theory and application}

As mentioned in Introduction the model proposed is based first of all on the S-ERAS model equations [1, 2, 5]. All changes concern the chemical part (the physical part remains unchanged) where the excess molar functions are expressed using the concentration of free and bounded segments (or bonds) [3]. The relationship between the free and bounded segments fulfills the relation

$$
c_{i}=z_{i}\left(1+\sum_{j} K_{i j} z_{j}\right),
$$

where $c_{i}$ is the concentration of stoichiometric species, $z_{i}$ - concentration of free segments, $K_{i j} z_{j}$ - concentrations of bounded segments or bonds, $K_{i j}$ - association constant.

The chemical contribution to the excess molar enthalpy may be expressed as

$$
\begin{aligned}
& H_{\mathrm{chem}}^{\mathrm{E}} / r_{\mathrm{m}}=\Delta h_{A}^{*} K_{A A}\left(z_{A}^{2}-\phi_{A} z_{A}^{02}\right)+\Delta h_{B}^{*} K_{B B}\left(z_{B}^{2}-\phi_{B} z_{B}^{02}\right) \\
& \quad+2 \Delta h_{A B} K_{A B} z_{A} z_{B}
\end{aligned}
$$


and to the excess molar volume as

$$
\begin{aligned}
& V_{\text {chem }}^{\mathrm{E}} / r_{\mathrm{m}}=\Delta v_{A}^{*} K_{A A}\left(z_{A}^{2}-\phi_{A} z_{A}^{02}\right)+\Delta v_{B}^{*} K_{B B}\left(z_{B}^{2}-\phi_{B} z_{B}^{02}\right) \\
& \quad+2 \Delta v_{A B} K_{A B} z_{A} z_{B},
\end{aligned}
$$

where $r_{\mathrm{m}}$ is the molar number of stoichiometric species, $\Delta v_{i j}^{*}$ - the molar volume change of creating hydrogen bonds of the $i-j$ type, $\Delta h_{i j}$ - the enthalpy of creating hydrogen bonds, $\phi_{i}$ - stoichiometric volume fraction, the superscript 0 refers to the pure component.

For all calculations (at $298.15 \mathrm{~K}$ ) the association parameters of pure alcohols: $K_{i j}, \Delta h_{i}^{*}$ and other required parameters were taken from the original ERAS model $[1,5]$. The experimental excess molar enthalpies and excess molar volumes were taken from the literature [6-11].

In the first part, the testing was performed for the an alkane + alcohol mixture in order to check the compatibility between the proposed approach and the original S-ERAS model [2]. As the first step, the energetic parameter, $X_{A B}$ was fitted to the experimental excess molar enthalpies of the hexane + hexan-1-ol mixture [7], and then the value of $\Delta v_{i}^{*}$ was adjusted to the excess volumes of the same system [6].

The results of calculations are presented in Fig. 1. The comparison of fitting parameters obtained using S-ERAS model with those from the application of the new modification of S-ERAS model, together with the percent deviations of $V^{\mathrm{E}}$ and $H^{\mathrm{E}}$, are given in Table I.
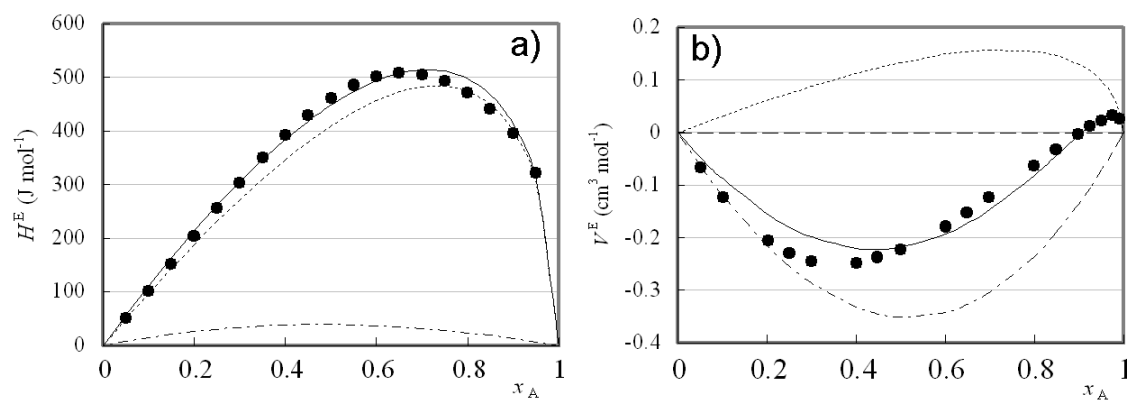

Fig. 1. Results of application of modified S-ERAS model to the description of excess molar enthalpies (a) and excess molar volumes (b) of hexane + hexan-1-ol mixture at 298.15 K; points - experimental values taken from literature [6, 7], solid line - model, dotted line - chemical contribution to excess molar function, dotted-dashed line physical contribution to excess molar function, $x_{A}$ is a mole fraction of hexane.

In the second part, the ability of the new model to describe simultaneously the excess molar enthalpies and excess molar volumes of pentan-1-ol + decan-1-ol and octan-1-ol + butan-1-ol mixtures was examined. Both thermodynamic quanties were reproduced independently using the assumptions: $\Delta v_{A}^{*}=\Delta v_{B}^{*}=$ 
TABLE I

Comparison of fitting parameters obtained using the S-ERAS model and modified S-ERAS model for description of excess molar enthalpies and prediction of excess molar volumes of hexane + hexan-1-ol mixture.

\begin{tabular}{c|c|c}
\hline \hline Parameters & SERAS & Modified model \\
\hline \multicolumn{2}{c}{ hexane + hexan-1-ol } \\
\hline$X_{A B}\left[\mathrm{~J} \mathrm{~cm}^{-3}\right]$ & 3.84 & 3.82 \\
$\Delta v_{B}\left[\mathrm{~cm}^{3} \mathrm{~mol}^{-1}\right]$ & -7.26 & -8.20 \\
$\delta H^{\mathrm{E}}[\%]$ & 3.0 & 3.0 \\
$\delta V^{\mathrm{E}}[\%]$ & 14.2 & 15.7
\end{tabular}
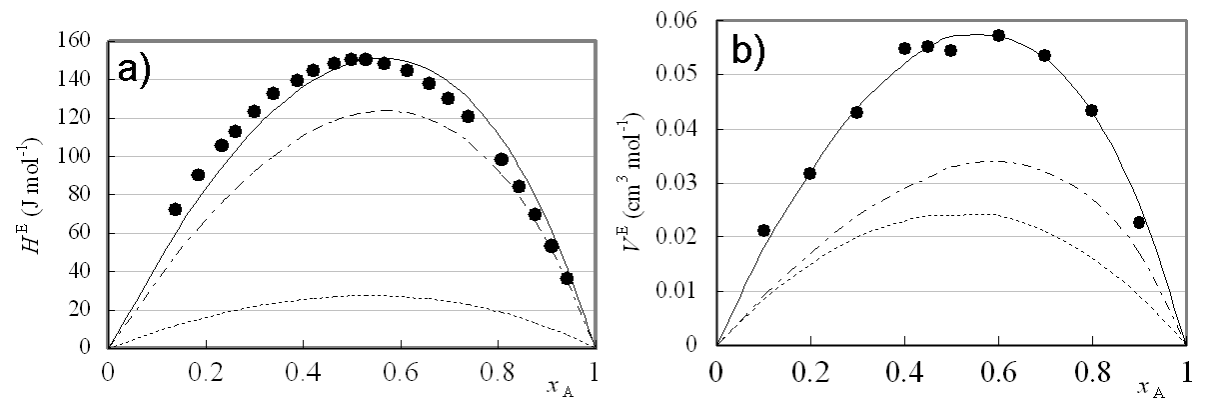

Fig. 2. Results of application of modified S-ERAS model to the description of excess molar enthalpies (a) and excess molar volumes (b) of pentan-1-ol + decan-1-ol mixture at 298.15 K; points - experimental values taken from literature [8, 9], solid line - model, dotted line - chemical contribution to excess molar function, dotted-dashed line physical contribution to excess molar function, $x_{A}$ is a mole fraction of pentan-1-ol.

$\Delta v_{A B}^{*}=-5.6 \mathrm{~cm}^{3} / \mathrm{mol}, \Delta h_{A}^{*}=\Delta h_{B}^{*}=\Delta h_{A B}^{*}=-25.1 \mathrm{~kJ} / \mathrm{mol}$ and the geometric mean rule for cross-association constant $\left(K_{A B}=\sqrt{K_{A} K_{B}}\right)$. In this way, the only fitting parameter was the energetic one, $X_{A B}$. The results are presented in Figs. 2 and 3 . The fitting parameters and the percent deviations of excess molar quantities are collected in Table II.

Illustration of the calculations made for hexane + hexan-1-ol mixture (Fig. 1 and Table I) confirms that the changes in the chemical part of the S-ERAS model affect the results of the description of excess molar quantities only slightly. However, for the better compatibility, the effect of contractions caused by creation of hydrogen bonds should be taken into consideration in Eq. (1).

In our first attempt, the ability of the new model to describe the excess molar enthalpies and excess molar volumes of two binary mixtures of alcohols was confirmed (Figs. 2 and 3, Table II). It should be emphasized that such description 

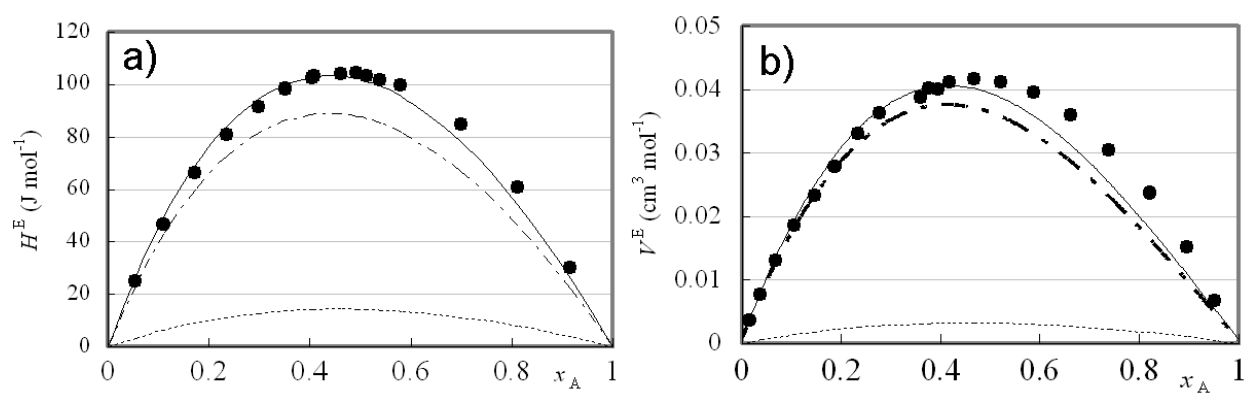

Fig. 3. Results of application of modified S-ERAS model to the description of excess molar enthalpies (a) and excess molar volumes (b) of octan-1-ol + butan-1-ol mixture at $298.15 \mathrm{~K}$; points - experimental values taken from literature [10, 11], solid line model, dotted line - chemical contribution to excess molar function, dotted-dashed line - physical contribution to excess molar function, $x_{A}$ is a mole fraction of octan-1-ol.

\section{TABLE II}

Fitting parameters obtained using the modified S-ERAS model for description of excess molar enthalpies and excess molar volumes of pentan-1-ol + decan-1-ol and octan-1-ol + butan-1-ol mixtures.

\begin{tabular}{c|c|c}
\hline \hline & \multicolumn{2}{|c}{ Quantity described } \\
\hline Parameters & $H^{\mathrm{E}}$ & $V^{\mathrm{E}}$ \\
\hline \multicolumn{2}{c}{ pentan-1-ol + decan-1-ol } \\
\hline$X_{A B}\left[\mathrm{~J} \mathrm{~cm}^{-3}\right]$ & 4.13 & 3.95 \\
$\delta X^{\mathrm{E}}[\%]$ & 6.4 & 3.3 \\
\hline \multicolumn{2}{c}{ octan-1-ol + butan-1-ol } \\
\hline$X_{A B}\left[\mathrm{~J} \mathrm{~cm} \mathrm{~cm}^{-3}\right]$ & 3.59 & 4.47 \\
$\delta X^{\mathrm{E}}[\%]$ & 3.3 & 7.0
\end{tabular}

was not possible using the traditional S-ERAS model without an enormous increasing $\Delta v_{i j}^{*}$ and $\Delta h_{i j}^{*}$ values. Also the cross association constants were probably too small to be acceptable from the physical point of view [12]. On the whole, the model proposed in this work enables the correct description or prediction of excess molar volumes and excess molar enthalpies providing that the relative magnitude of physical and chemical contributions to regarded excess molar quantities will be established. It seems that the best way to characterize the physical contribution is finding the proper reference mixtures (containing the homomorphs of alcohols) in which the association phenomena do not occur. 


\section{References}

[1] A. Heintz, Ber. Bunsenges. Phys. Chem. 89, 172 (1985).

[2] A. Pineiro, A. Amigo, R. Bravo, P. Brocos, Fluid Phase Equilib. 173, 211 (2000).

[3] T. Vasiltsova, A. Heintz, J. Chem. Phys. 127, 114501 (2007).

[4] S.W. Campbell, Fluid Phase Equilib. 102, 61 (1994).

[5] M. Bender, A. Heintz, Fluid Phase Equilib. 89, 197 (1993).

[6] A.J. Treszczanowicz, G.C. Benson, Int. Data Series, Selected Data Mixtures, Ser. A 4, 255 (1986).

[7] A. Heintz, B. Schmittecker, D. Wagner, R.N. Lichtenthaler, J. Chem. Eng. Data 31, 487 (1986).

[8] H.H. Sun, J.J. Christensen, R.M. Izatt, R.W. Hanks, J. Chem. Thermodyn. 12, 95 (1980).

[9] M. Gepert, B. Stachowska, J. Solution Chem. 35, 425 (2006).

[10] A.E. Pope, H.D. Pflug, B. Dacre, G.C. Benson, Can. J. Chem. 45, 2665 (1967).

[11] H.D. Pflug, G.C. Benson, Can. J. Chem. 46, 288 (1968).

[12] M. Gepert, B. Hachuła, J. Mol. Liq. 135, 196 (2007). 